\title{
K band monitoring of GRS 1915+105 during 1999/2000
}

\author{
J. Greiner \\ Astrophysical Institute Potsdam, 14482 Potsdam, Germany
}

F.J. Vrba, A.A. Henden, H.H. Guetter and C.B. Luginbuhl

US Naval Observatory, Box 1149, Flagstaff Station, Flagstaff, AZ 86002, USA

\begin{abstract}
We report on long-term $\mathrm{K}$ band photometry of the galactic microquasar GRS 1915+105 since March 1999. We see variations of more than 1 mag, which are seemingly correlated with variations in X-ray intensity and spectral slope.
\end{abstract}

Keywords: X-ray binary, GRS 1915+105, infrared observations

\section{Introduction}

GRS $1915+105$ is the prototypical microquasar, a galactic X-ray binary ejecting plasma clouds at $\mathrm{v} \approx 0.92 \mathrm{c}$ (Mirabel \& Rodriguez 1994). Three different sources of infrared (IR) radiation are expected from a X-ray binary, namely thermal emission from the outer part of accretion disk, thermal emission from the companion star or synchrotron emission from the jet(s). Previous IR observations of GRS 1915+105 include, besides a few single measurements since 1993, some few hrs photometry in conjunction with X-ray/radio measurements (Eikenberry et al. 2000), and a 2 month monitoring in 1996 (Bandyopadhyay et al. 1998).

\section{Observations and Results}

$\mathrm{K}$ band observations have been performed with the 1.55-m telescope of the US Naval Observatory Flagstaff equipped with the $256 \times 256$ pixel HgCdTe IRCAM camera during 8-10 day intervals around full Moon. Because the main interest was in the long-term behaviour, "only" about 1-3 observations per month were done, consisting of groups of 3 or 4 individual observations per night. Each group represents a set of coadded integrations with an exposure of $400 \mathrm{sec}$. In total, observations were performed on 34 nights between 05 May 1999 and 15 Jun 2000.

These observations show GRS $1915+105$ to be variable between 12.1 $\lesssim \mathrm{K} \lesssim 13.7 \mathrm{mag}$ (not extinction-corrected; Fig. 1). Our long coverage allows to search for correlations with other emission characteristics, e.g. the RXTE All-Sky Monitor (ASM) quick-look results provided by the ASM/RXTE team. After selecting those $\mathrm{K}$ band observations which

(c) 2020 Kluwer Academic Publishers. Printed in the Netherlands. 




Figure 1. K band light curve of GRS 1915+105, showing the mean of all "groups".

were done within $4 \mathrm{hrs}$ of an ASM scan, we find that both the X-ray flux as well as the X-ray hardness ratio (flux ratio of $5-12 \mathrm{keV}$ vs. 3-5 $\mathrm{keV}$ band) are positively correlated with the $\mathrm{K}$ band flux.

A regression analysis suggests that a doubling of the X-ray count rate (flux) leads to a $33 \%$ increase in the $\mathrm{K}$ band flux. This argues against an explanation due to illumination of the secondary. Instead, it rather could be synchrotron radiation related to (small) ejections/flares as seen also at shorter time scales (Eikenberry et al. 2000). In this picture the correlation of the $\mathrm{K}$ band flux with the X-ray hardness ratio is compatible with the correlation of X-ray spectral slope and radio flux found by Rau \& Greiner (2001). A large (small) value of the hardness ratio corresponds to a flat (steep) X-ray power law slope, and therefore a high (low) temperature of the electrons in the corona if the general comptonization paradigm is adopted. These correlations then imply that strong $\mathrm{K}$ band flux and weak radio flux occur during times of a flat X-ray power law, i.e. most of the synchrotron power is radiated either in the infrared or radio band depending only on the temperature of electrons in the corona.

\section{References}

Bandyopadhyay R., Martini P., Gerard E., et al. 1998, MNRAS 295, 623

Eikenberry S.S., Matthews K., Muno M., et al. 2000, ApJ (astro-ph/0001472)

Mirabel I.F., Rodriguez L.F.: 1994, Nature 371, 46

Rau A., Greiner J., 2001, these proceedings 